\title{
TCM, TTCM, BICM AND ITERATIVE BICM ASSISTED OFDM-BASED DIGITAL VIDEO BROADCASTING TO MOBILE RECEIVERS
}

\author{
C.S. Lee, S.X. Ng, L. Piazzo, L. Hanzo \\ Dept. of Electr. and Comp. Sc., Univ. of Southampton, SO17 1BJ, UK. \\ Tel: +44-23-80593125, Fax: +44-23-80594508 \\ Email: lh@ecs.soton.ac.uk \\ http://www-mobile.ecs.soton.ac.uk
}

\begin{abstract}
In this contribution, the performance of trellis coded modulation (TCM), bit-interleaved coded modulation (BICM), BICM with iterative decoding (BICM-ID) and turbo trellis coded modulation (TTCM) is studied in comparison to the pan-European terrestrial Digital Video Broadcasting (DVB) system. The decoding complexity and bandwidth efficiency trade-offs of the various systems under study are quantified.
\end{abstract}

\section{INTRODUCTION}

Since the invention of trellis coded modulation (TCM) in the 1970s by Ungerboeck and Csajka [1, 2], coded modulation schemes have been popular since they achieve coding gain without bandwidth expansion. The first application of TCM can be found in the area of digital transmission over telephone lines, notably in the International Telecommunication Union's (ITU) 9.6/14.4 kbps trellis coded modems [3]. TCM has also been found to be suitable in mobile communications scenarios $[4,5]$, where bandwidth efficiency is at a premium. Turbo trellis coded modulation (TTCM) [6] has a structure similar to that of the family of power efficient turbo codes [7], but utilises TCM codes as its constituent codes. Both schemes employ set partitioning based signal labelling, in order to increase the minimum Euclidean distance between the encoded information bits.

A more recently discovered member in the coded modulation family is Bit Interleaved Coded Modulation (BICM), which uses Gray-coded signal labelling [8]. It combines conventional convolutional codes with several independent bit interleavers. The number of bit interleavers equals the number of bits represented by a modulation symbol. Each bit interleaver is independent of the other bit interleavers.

Recently, Li and Ritcey proposed a BICM enhancement employing iterative decoding $[9,10,11]$, where

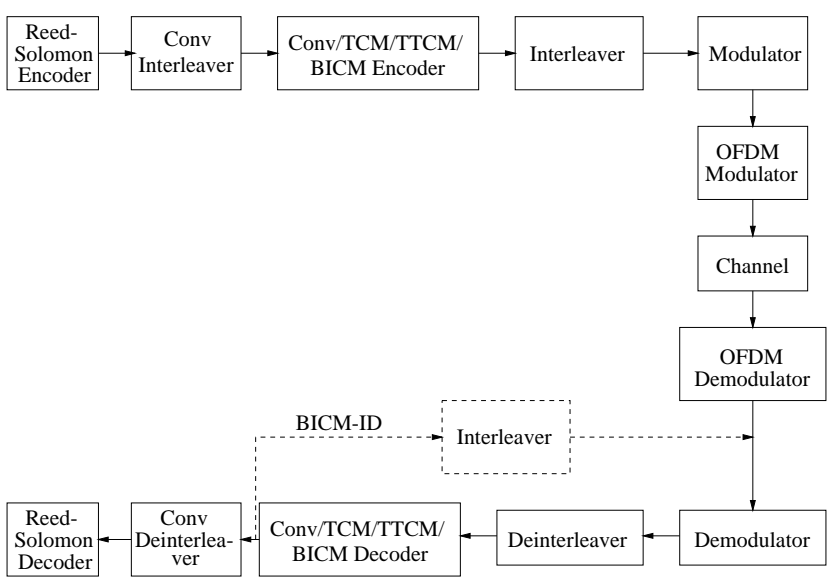

Figure 1: Block diagram of the different coded modulation systems.

the BICM decoder feeds its soft output metrics back to the input of the demodulator a number of times, before hard decision is invoked. The signal mapping is based on set partitioning.

The aim of our work is to study the decoding complexity and bandwidth efficiency trade-offs struck by these coded modulation schemes in comparison to the pan-European terrestrial DVB system [12, 3, 13]. We are particularly interested in comparing the performance of these coded modulation schemes and the convolutional coded terrestrial DVB scheme [12]. In the DVB standard [12], the convolutional code acts as the inner code, which is concatenated to an outer Reed-Solomon code [14].

\section{SYSTEM OVERVIEW}

The block diagram of the coded modulation schemes used in this study is shown in Figure 1. Pseudo-random source bits are generated and fed into the Reed-Solomon (RS) encoder. The RS encoder forms a concatenated 
coding scheme with one of the inner channel encoders, namely the convolutional, TCM, BICM or TTCM encoder. The RS encoder adds 16-byte of parity symbols to the 188-byte input symbols in order to correct eight erroneous bytes for each 204-byte packet. The convolutional interleaver adopted in this study is defined in the terrestrial DVB standard [12]. The coded bitstream is interleaved prior to being mapped to the modulated symbols based on their respective mapping strategies. For convolutional and BICM encoding, the mapping is based on Gray labelling. On the other hand, TCM, TTCM and BICM-ID use set partitioning based mapping of the bits to modulated symbols. In order to counteract the effects of dispersive multipath fading channels, we have adopted the DVB standard's Orthogonal Frequency Division Multiplexing (OFDM) scheme [3]. The parameters of the OFDM DVB system are presented in Table 1, while those of the convolutional encoder are summarised in Table 2.

\begin{tabular}{|l|r|}
\hline OFDM Parameters \\
\hline Total number of subcarriers & $2048(2 \mathrm{~K}$ mode $)$ \\
Number of effective subcarriers & 1705 \\
OFDM symbol duration $T_{s}$ & $224 \mu \mathrm{s}$ \\
Guard Interval & $T_{s} / 4=56 \mu \mathrm{s}$ \\
Total symbol duration & $280 \mu \mathrm{s}$ \\
(inc. Guard Interval) & \\
Consecutive subcarrier spacing $1 / T_{s}$ & $4464 \mathrm{~Hz}$ \\
DVB channel spacing & $7.61 \mathrm{MHz}$ \\
QPSK and QAM symbol period & $7 / 64 \mu \mathrm{s}$ \\
\hline
\end{tabular}

Table 1: Parameters of the OFDM module [12].

\begin{tabular}{|l|c|}
\hline \multicolumn{2}{|l|}{ Convolutional Coder Parameters } \\
\hline Code Rate & $1 / 2$ \\
Constraint Length & 7 \\
$n$ & 2 \\
$k$ & 1 \\
Generator Polynomials (octal format) & 171,133 \\
\hline
\end{tabular}

Table 2: Parameters of the $\mathrm{CC}(n, k, K)$ convolutional inner encoder of the DVB terrestrial modem [12].

Table 3 shows the generator polynomials of the TCM and TTCM codes, which are presented in octal format. These codes are systematic codes and the encoder attaches only one parity bit to the information bits. Hence, the code rate of the $2^{m+1}$-ary signal is $R=\frac{m}{m+1}$.

Table 4 shows the BICM and BICM-ID codes' generator polynomials in octal format. These are nonsystematic codes, which also produces one parity bit only. Hence, the code rates of these codes are similar to those

\begin{tabular}{|c|l|c|c|c|c|c|}
\hline Code Rate & State & $m$ & $H^{0}$ & $H^{1}$ & $H^{2}$ & $H^{3}$ \\
\hline \hline $1 / 2$ & 8 & 1 & 11 & 02 & - & - \\
(QPSK) & & & & & & \\
\hline $2 / 3$ & 8 & 2 & 11 & 02 & 04 & - \\
$(8 \mathrm{PSK})$ & 16 & 2 & 23 & 02 & 10 & - \\
& $128 *$ & 2 & 277 & 54 & 122 & - \\
\hline $3 / 4$ & 8 & 3 & 11 & 02 & 04 & 10 \\
$(16 \mathrm{QAM})$ & 16 & 3 & 21 & 02 & 04 & 10 \\
\hline
\end{tabular}

Table 3: Summary of the TCM and TTCM constituent codes proposed by Ungerboeck [2] as well as Robertson and Wörz [6], where $m$ refers to the number of coded information bits. The code generator polynomial, $H^{i}$, is presented in octal format. The ' $*$ ' symbol refers to Ungerboeck's code.

\begin{tabular}{|c|l|l|l|l|l|}
\hline Code Rate & State & $g^{0}$ & $g^{1}$ & $g^{2}$ & $g^{3}$ \\
\hline \hline $1 / 2$ & 8 & 15 & 17 & - & - \\
(QPSK) & & & & & \\
\hline $2 / 3$ & 8 & 4 & 2 & 6 & - \\
$(8 \mathrm{PSK})$ & & 1 & 4 & 7 & - \\
\cline { 2 - 6 } & 16 & 7 & 1 & 4 & - \\
& & 2 & 5 & 7 & - \\
\hline $3 / 4$ & 8 & 4 & 4 & 4 & 4 \\
$(16 \mathrm{QAM})$ & & 0 & 6 & 2 & 4 \\
& & 0 & 2 & 5 & 5 \\
\hline
\end{tabular}

Table 4: Summary of the convolutional codes employed in the BICM encoder. These codes were obtained from page 331 of [15]. The code generator polynomial, $g^{i}$, is presented in octal format.

of the TCM and TTCM codes seen in Table 3 .

The DVB terrestrial convolutional code uses soft decision Viterbi decoding, while the TCM, TTCM and BICM codes invoke the Maximum A-Posteriori (MAP) [16] decoding algorithm.

\section{CHANNEL MODEL}

The channel model employed in this study was the twelve-path COST 207 [17] Hilly Terrain (HT) type impulse response, with a maximal relative path delay of $19.9 \mu \mathrm{s}$. This channel was selected, in order to provide a worst-case propagation scenario for the DVB-T system employed in our study, upon using a mobile receiver.

In the system characterised here, we have used a carrier frequency of $500 \mathrm{MHz}$ and a sampling rate of $7 / 64 \mu \mathrm{s}$. Each of the channel paths was faded independently obeying a Rayleigh fading distribution, according to a normalised Doppler frequency of $10^{-5}$ [14]. This corresponds to a worst-case vehicular velocity of 


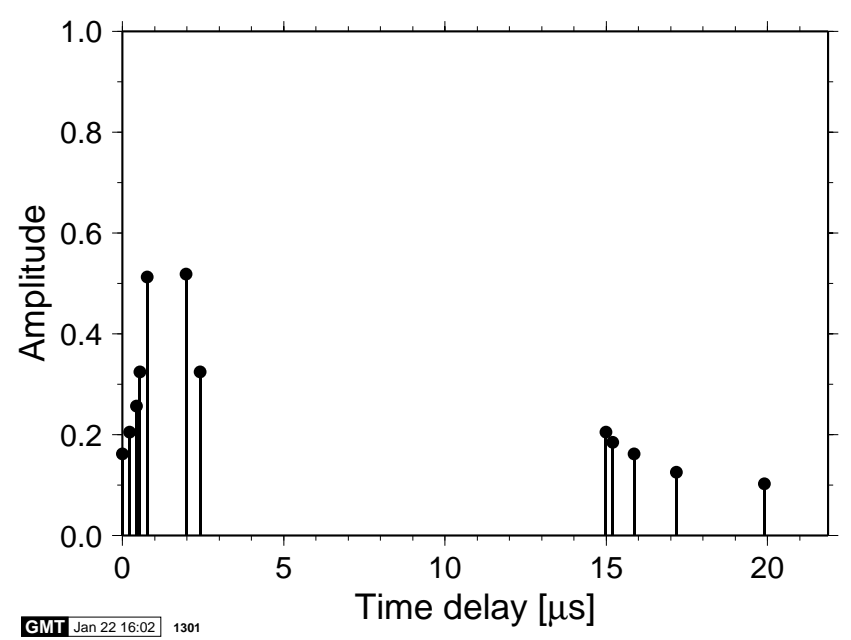

Figure 2: COST 207 Hilly Terrain (HT) type impulse response [17].

about $200 \mathrm{~km} / \mathrm{h}$. The unfaded impulse response is depicted in Figure 2. For the sake of completeness we note that the standard COST 207 channel model was originally defined in order to facilitate the comparison of different GSM implementations [14] under standard conditions. The associated GSM bit rate was $271 \mathrm{kbit} / \mathrm{s}$. By contrast, in our investigations the bit rate of DVB-quality transmissions can be as high as 20 Mbit/s, where there is a higher number of resolvable multipath components within the dispersion-range considered, than at the GSM rate of $271 \mathrm{kbit} / \mathrm{s}$. However, the performance of various wireless tranceivers is well understood by the research community over this standard COST 207 channel and hence its employment is beneficial in benchmarking terms. Furthermore, since the OFDM modem has 2048 subcarriers, the subcarrier signalling rate is effectively 2000-times lower than our maximum DVB-rate of $20 \mathrm{Mbit} / \mathrm{s}$, corresponding to $10 \mathrm{kbit} / \mathrm{s}$. At this subchannel rate, the individual subchannel can be considered nearly frequency-flat. In summary, in conjunction with the $200 \mathrm{~km} / \mathrm{h}$ vehicular speed the investigated channel conditions constitute a pessimistic scenario.

\section{SIMULATION RESULTS AND DISCUSSIONS}

In this section, first we compare the performance of the coded modulation schemes to that of the DVB terrestrial system using the rate $1 / 2$ inner code. Specifically, Figure 3 shows the performance of the rate $1 / 2$ TCM, BICM, BICM-ID and DVB-T convolutional coding schemes over the wideband fading channel of Figure 2. WE note, however that these schemes have different decoding complexities. The rate $1 / 2$ TCM code

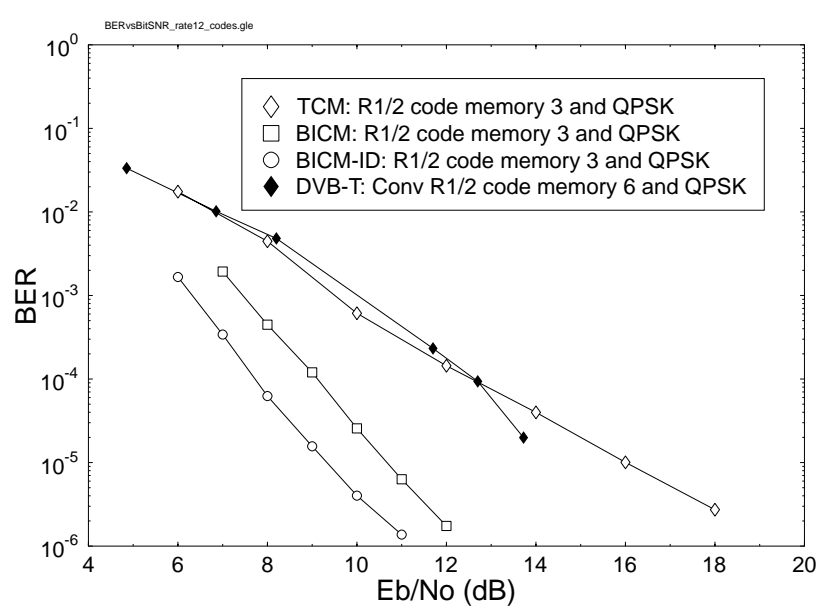

Figure 3: Performance of the rate 1/2 TCM, BICM, BICM-ID and DVB-T convolutional coding schemes having a different decoding complexity over the wideband fading channel of Figure 2. All schemes have a useful throughput of $1 \mathrm{bit} / \mathrm{s} / \mathrm{Hz}$.

is shown to perform similarly to the DVB-T rate $1 / 2$ convolutional code. However, the TCM scheme is less complex, since its code memory is only half that of the convolutional code. The convolutional code begins to show coding gain over the rate $1 / 2$ TCM scheme, when the bit energy exceeds $12.7 \mathrm{~dB}$ in this case. On the other hand, the BICM and BICM-ID schemes not only exhibit a lower decoding complexity, than the convolutional code, but also achieve coding gains of $3.5 \mathrm{~dB}$ and $4.9 \mathrm{~dB}$, respectively, at a BER of $10^{-4}$.

Figure 4 shows the performance of the TCM schemes, over the dipersive fading channel of Figure 2 when employing rate $2 / 3$ codes using 8 -state, 16 -state and 64 state trellises. Again, we use the rate 1/2 and 3/4 convolutional codes employed in the DVB terrestrial system as benchmarkers. The performance of the 8-state and 16 -state rate $2 / 3 \mathrm{TCM}$ codes lies in between the rate $1 / 2$ and rate $3 / 4$ convolutional codes' performance. This implies that that these rate $2 / 3$ TCM codes perform over the dispersive fading channel of Figure 2 similarly to the rate $2 / 3$ convolutional code adopted by the DVB standard (not shown in the graph). Hence, these TCM codes can achieve the same performance as the convolutional codes, but at a lower computational complexity. This observation was also valid for the rate $1 / 2$ TCM scheme. When the code memory of the rate $2 / 3$ TCM code was increased to 7 , the associated performance was similar to that of the 64-state rate $1 / 2$ convolutional code. Furthermore, we note that this TCM scheme transmitted using 8PSK modulation, which carries two information bits and one parity bits. On the other hand, the rate $1 / 2$ convolutional codec of 


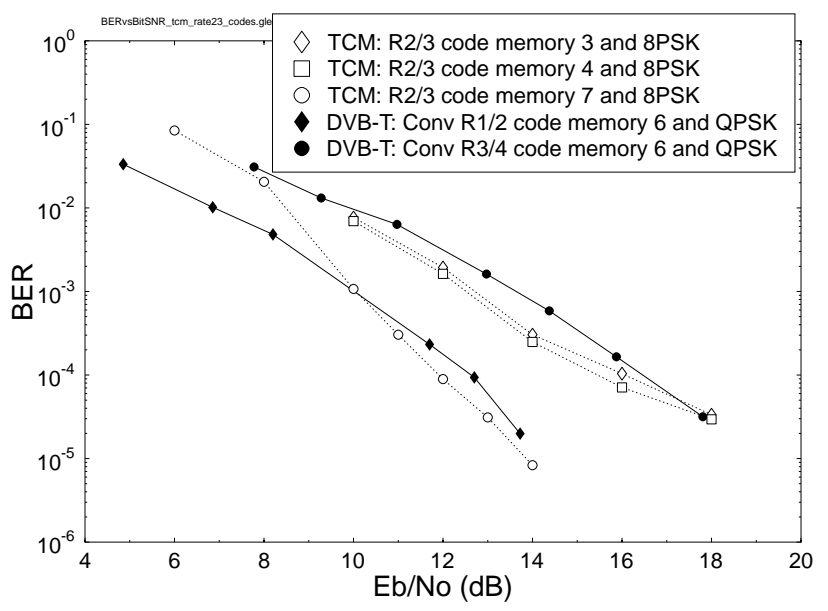

Figure 4: Performance of rate $2 / 3$ TCM in comparison to the rate $1 / 2$ and $3 / 4$ DVB-T convolutional codes exhibiting different decoding complexity for transmissions over the wideband fading channel of Figure 2. The coded modulation scheme has throughput of 2 bits/s/Hz. The DVB scheme employing rate $1 / 2$ convolutional code and QPSK modulation has throughput of $1 \mathrm{bit} / \mathrm{s} / \mathrm{Hz}$, while the rate $3 / 4$ inner code case has throughput of $1.5 \mathrm{bit} / \mathrm{s} / \mathrm{Hz}$.

the DVB system transmitted using QPSK modulation, which carries one information bit and one parity bit within the same bandwidth. Therefore, the rate $2 / 3$ TCM code is capable of transmitting at twice the useful bitrate compared to the DVB system for the same bandwidth and similar codec complexity.

Let us now compare the performance of the rate 2/3 BICM and BICM-ID codes to that of the DVB convolutional codes, which is shown in Figure 5. The 8 -state and 16 -state rate $2 / 3$ BICM codes perform similarly to the 64 -state rate $1 / 2$ convolutional code. When the code complexity of the rate $2 / 3 \mathrm{BICM}$ code is increased to 64 states, it achieves an effective bandwidth efficiency of $2 \mathrm{bits} / \mathrm{s} / \mathrm{Hz}$, while also providing a coding gain of $1.3 \mathrm{~dB}$ over the 64 -state rate $1 / 2$ convolutional code, which only has a bandwidth efficiency of $1 \mathrm{bit} / \mathrm{s} / \mathrm{Hz}$. The 8 -state and 16 -state rate $2 / 3$ BICMID codes exhibit an even better performance, as shown in Figure 5. Apart from possessing better spectral efficiency than the rate $1 / 2$ convolutional code, the 8 -state rate $2 / 3$ BICM-ID code requires only half the decoding complexity, while exhibiting a coding gain of $2.4 \mathrm{~dB}$ at a BER of $10^{-4}$.

We continued our evaluation of the coded modulation schemes' performance using rate $3 / 4$ codes. The 8 -state and 16-state rate $3 / 4$ TCM schemes' performance is similar to the 64 -state rate $3 / 4$ convolutional code, but at a lower decoding complexity. However,

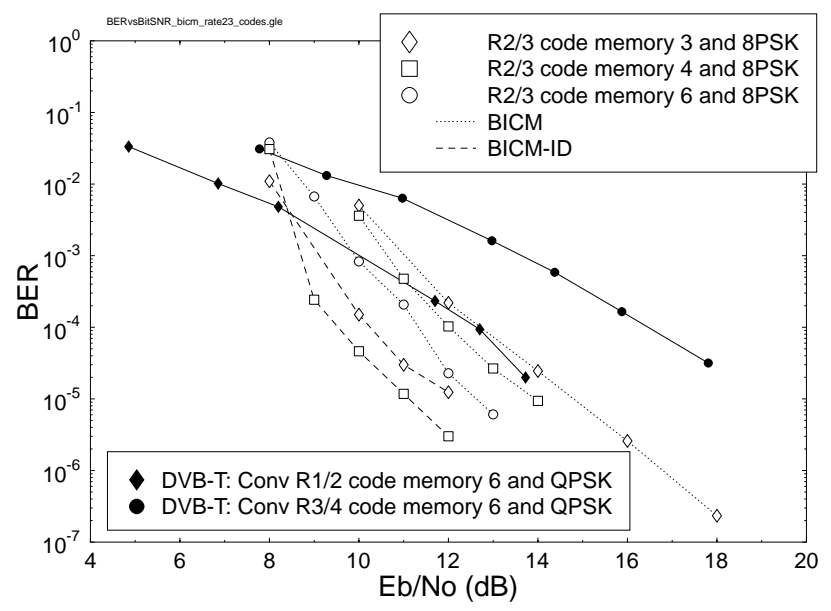

Figure 5: Performance of rate $2 / 3$ BICM and BICM-ID in comparison to the rates $1 / 2$ and $3 / 4$ DVB-T convolutional codes exhibiting different decoding complexity for transmissions over the wideband fading channel of Figure 2. The coded modulation scheme has throughput of $2 \mathrm{bits} / \mathrm{s} / \mathrm{Hz}$. The DVB scheme employing rate $1 / 2$ convolutional code and QPSK modulation has throughput of $1 \mathrm{bit} / \mathrm{s} / \mathrm{Hz}$, while the rate $3 / 4$ inner code case has throughput of $1.5 \mathrm{bit} / \mathrm{s} / \mathrm{Hz}$.

when the rate $3 / 4 \mathrm{BICM}$ code having a memory of 3 is employed, it exhibits a coding gain of $5.4 \mathrm{~dB}$ at a BER of $10^{-4}$ over the rate $3 / 4$ convolutional code. We can attain further coding gain, if we employ the 8-state rate $3 / 4$ BICM-ID code, which increases the coding gain by an additional $2.1 \mathrm{~dB}$.

Figure 7 shows the performance of the coded modulation systems, when TTCM is employed. The rate 1/2 TTCM having a code memory 3 constituent TCM scheme, is seen to perform on par with the rate $1 / 2$ turbo code, which we proposed in [13] as a replacement for the convolutional code. However, the TTCM code employs a smaller interleaver size of 8000 bits, instead of the 17952 bit memory of the turbo code's interleaver. This is equivalent to a potential saving of $60 \%$ of storage space and latency. The rate $2 / 3$ TTCM scheme's performance is observed to be between that of the DVB system's employing QPSK and 64QAM modulation modes using rate $1 / 2$ turbo codes. This indirectly implies that the rate $2 / 3$ TTCM scheme exhibits similar performance characteristics to the 16QAM DVB system employing the same rate $1 / 2$ turbo code. From Figure 7 , we also observe that the rate $3 / 4$ TTCM code has a bandwidth efficiency gain of $50 \%$ in comparison to that of the rate $1 / 2$ turbo coded DVB-like system utilising 64QAM modulation. Furthermore, it also exhibits a coding gain of $2.1 \mathrm{~dB}$ at a BER of $10^{-4}$. 


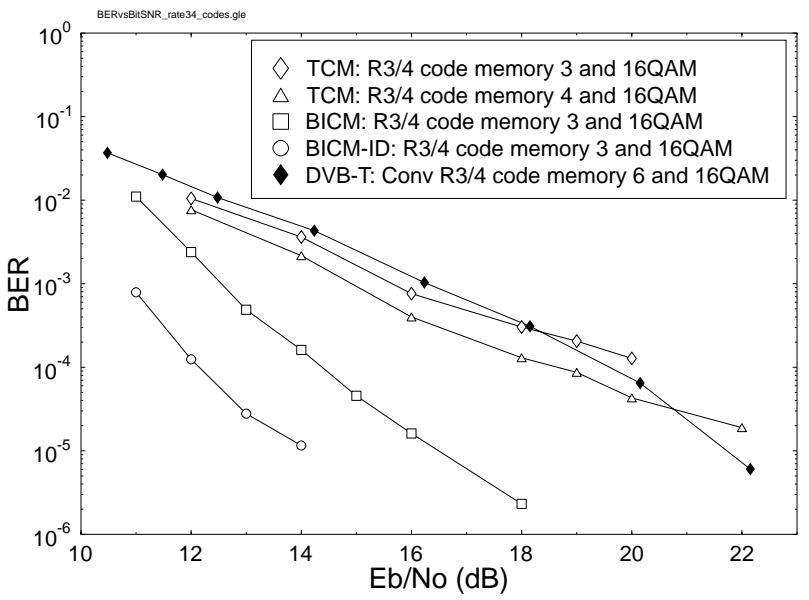

Figure 6: Performance of rate 3/4 TCM, BICM, BICMID and the DVB-T convolutional codes at different decoding complexity over the wideband fading channel of Figure 2. All schemes have a useful throughput of 3 $\mathrm{bit} / \mathrm{s} / \mathrm{Hz}$.

\section{SUMMARY}

In this contribution, we have studied coded modulation schemes for transmissions over the COST 207 dispersive wideband channel of Figure 2. The TCM schemes can attain a performance similar to that of the convolutional codes adopted by the DVB standard, albeit at a lower decoding complexity. The BICM codes consistently outperformed the convolutional code at the same code rate. The coding gain of BICM can be further increased, if we employ iterative decoding. Comparing the four coded modulation schemes, the TTCM ssystems provide the best performance in terms of power efficiency. In higher-order constellation modes, the TTCM scheme is seen to have a better spectral efficiency, than the turbo coded DVB-like system. However, in loworder constellation modes their spectral efficiency is similar.

\section{REFERENCES}

[1] G. Ungerboeck and I. Csajka, "On improving data link performance by increasing the channel alphabet and introducing sequence coding," in International Symposium on Information Theory, (Ronneby, Sweden), June 1976.

[2] G. Ungerboeck, "Channel coding with multilevel/phase signals," IEEE Transactions on Information Theory, vol. IT-28, pp. 55-67, January 1982.

[3] L. Hanzo, W. Webb, and T. Keller, Single and Multicarrier Modulation: For Personal Communications, WLANs and Broadcasting. John Wiley \& Sons, 2000.

[4] D. Divsalar and M. K. Simon, "The design of trellis coded MPSK for fading channel: Performance criteria," IEEE Transactions on Communications, vol. 36, pp. 1004-1012, September 1988.

[5] D. Divsalar and M. K. Simon, "The design of trellis coded MPSK for fading channel: Set partitioning for optimum code design," IEEE Transactions on Communications, vol. 36, pp. 1013-1021, September 1988.

[6] P. Robertson and T. Wörz, "Bandwidth-efficient turbo trellis coded modulation using punctured component codes," IEEE

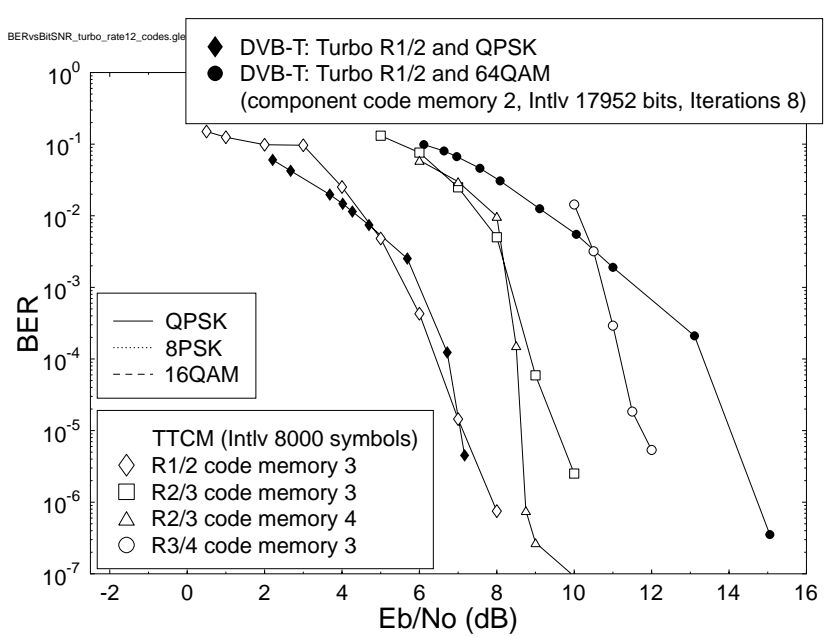

Figure 7: Performance of the TTCM scheme and the turbo coded DVB terrestrial system over the wideband fading channel of Figure 2. The turbo coded DVB schemes have useful throughput of $1 \mathrm{bit} / \mathrm{s} / \mathrm{Hz}$ and 3 $\mathrm{bit} / \mathrm{s} / \mathrm{Hz}$ for the QPSK and 64QAM modes respectively. The TTCM schemes have useful throughput of 1,2 and $3 \mathrm{bit} / \mathrm{s} / \mathrm{Hz}$ for the rate $1 / 2,2 / 3$ and $3 / 4$ case respectively.

Journal on Selected Areas in Communications, vol. 16, pp. 206-218, February 1998.

[7] C. Berrou, A. Glavieux, and P. Thitimajshima, "Near Shannon limit error-correcting coding and decoding: Turbo codes," in Proceedings of the International Conference on Communications, pp. 1064-1070, May 1993.

[8] E. Zehavi, "8-PSK trellis codes for a rayleigh channel," IEEE Transactions on Communications, vol. 40, pp. 873-884, May 1992.

[9] X. Li and J. A. Ritcey, "Bit-interleaved coded modulation with iterative decoding," IEEE Communications Letters, vol. 1, pp. 169-171, November 1997.

[10] X. Li and J. A. Ritcey, "Bit-interleaved coded modulation with iterative decoding using soft feedback," IEE Electronics Letters, vol. 34, pp. 942-943, May 1998.

[11] X. Li and J. A. Ritcey, "Trellis coded modulation with bit interleaving and iterative decoding," IEEE Journal on Selected Areas in Communications, vol. 17, pp. 715-724, April 1999.

[12] ETSI, Digital Video Broadcasting (DVB); Framing structure, channel coding and modulation for digital terrestrial television, August 1997. EN 300744 V1.1.2.

[13] C.S.Lee, T. Keller, and L. Hanzo, "OFDM-based turbo-coded hierarchical and non-hierarchical terrestrial mobile digital video broadcasting," IEEE Transaction on Broadcasting, vol. 46, pp. 1-22, March 2000.

[14] R. Steele and L. Hanzo, eds., Mobile Radio Communications: Second and Third Generation Cellular and WATM Systems. John Wiley \& Sons, 2 ed., 1999.

[15] S. Lin and D. J. Costello, Error Control Coding: Fundamentals and Applications. Prentice Hall, 1983.

[16] L. R. Bahl, J. Cocke, F. Jelinek, and J. Raviv, "Optimal decoding of linear codes for minimizing symbol error rate," IEEE Transactions on Information Theory, vol. 20, pp. 284-287, March 1974.

[17] M. Failli, "Digital land mobile radio communications COST 207," tech. rep., European Commission, 1989. 